\title{
THE BRIGHT ECLIPSING BINARY NSV 4031 IS NOT VARIABLE
}

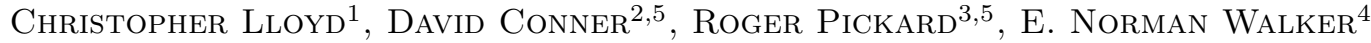 \\ 1) Department of Physics and Astronomy, University of Sussex, Brighton, BN1 9QH, UK C.Lloyd@sussex.ac.uk \\ 2) Somerby Observatory, Somerby, Melton Mowbray, LE14 2PP, UK dc@somerbyconners.plus.com
}

3) Shobdon Observatory, 3 The Birches, Shobdon, Leominster, HR6 9NG, UK

4) Deudneys Cottage, Old Road, Herstmonceux, Hailsham, BN27 1PU

5) BAA Variable Star Section, Burlington House, Piccadilly, London, W1J 0DU, UK

\begin{abstract}
Observations of the supposed eclipsing binary NSV 4031 from time-series photometry and data from several synoptic instruments show no evidence of an eclipse or any periodic variation with an amplitude above 0.05 magnitudes. The spectral energy distribution of the star is very closely matched to a spectral type of F5IV.
\end{abstract}

\section{Introduction}

NSV 4031 (HD 70271, BD +45¹570, CSV 6628, SVS 1396 Lyn, $082258.64+4527$ 23.7 J2000) is a ninth magnitude star in Lynx and was reported as a probable Algol-type eclipsing binary by Lange (1963). The report is very brief. There are no observations, no light curve and no description of what was actually seen. Visual observations made in the dusk of 1963 May 11, 12, 13, and 14 showed an apparent eclipse from 8.0 to 8.8 with a duration of 2.6 , and with an interval of 0.997 - effectively one sidereal day. Given the duration of the eclipse Lange derived a likely ephemeris of

$$
H J D_{\mathrm{MinI}}=2438162.297+0.4985 \times E
$$

It has to be said that there are some very curious aspects to this discovery. The observations were made in early May which is at the end of the observing season. Epoch zero of the ephemeris corresponds to 1963 May 12 19:08 UT and at this time the star was about 4 hours west of the meridian but still at $45^{\circ}$ altitude. On this day in Odesa sunset occurred at 17:19 UT and local astronomical twilight ended at 19:30 UT. With the eclipse lasting for 2 . 6 the fading part would have taken place from $\sim 30$ minutes after sunset until $\sim 20$ minutes before the end of twilight. It is not clear what Lange saw but most of the supposed eclipse must have taken place in the twilight. Although the visual range is quoted as 8.0 to 8.8 Tycho gives $V=9.2$ and similar magnitudes are reported here. Lange found that at maximum the variable was equal to $\mathrm{BD}+45^{\circ} 1565=\mathrm{HD} 69917$, for which Tycho gives $V=8.8$, and this discrepancy highlights the difficulty in making these observations. Lange has a long history of variable star discoveries but it is not clear what provoked his interest in this star or why he was observing it at this difficult time. There do not appear to be any previous or follow-up observations.

As a variable star NSV 4031 has been largely ignored but it was included in the BAA VSS Eclipsing Binary Programme (Loughney, 2015; Lloyd, 2018) in an effort to clarify 
Table 1: List of equipment used

\begin{tabular}{lcc}
\hline Observer & Telescope & CCD \\
\hline \hline Conner & BRT 64-mm f/2.8 lens & FLI MaxCam CM2-1 \\
Conner & Somerby 50-mm f/3 lens & Atik Titan Mono \\
Pickard & Shobdon LX 200 0.35-m SCT & SXVF-H9 \\
\hline
\end{tabular}

its nature. The programme is also described on the BAA VSS website. The star was mentioned by Isles (1989) but no observations seem to have been made at that time. A series of visual observations by T. Markham in 2003 February - March, has not been previously discussed but showed no significant variation with $m_{V}=9.6 \pm 0.2$. Photometry from a decade later is presented here in addition to data from a wide range of automatic survey telescopes that has not previously been analysed.

\section{Observations}

Observations were made during the 2012 - 2014 observing seasons specifically to test the variability of NSV 4031. A series of single and multiple measurements were made with the Bradford Robotic Telescope Cluster Camera (BRT) during both seasons and these were supplemented with additional time series runs using the Somerby 2-in Titan and Shobdon telescope during 2013 - 2014. The instruments are described in Table 1 and details of the multiple observations and time series runs are given in Table 2. All the data were dark-subtracted and flat-fielded prior to being measured using differential aperture photometry relative to an ensemble of comparison stars taken from a $V$-band sequence. There is a small offset between the two sets of BRT data and it is clear that the data from the first season are not well flat fielded. The BRT is not user controlled so flats and darks can change leading to differences in calibration, and this issue is know to occur in long sets of BRT data. The Shobdon telescope is rather large for a star of this magnitude and exposures had to be kept short to ensure that they were not saturated. Nevertheless these runs are much noisier than expected with $\sigma \sim 0$. 04 compared to $\sigma \sim 0{ }^{\mathrm{m}} 01$ for the Somerby runs, and points to some overexposure issues.

Additional data are taken from the archives of the synoptic survey projects Tycho, ROTSE-I, SuperWASP and ASAS-SN, but it has to be said that all these data are compromised to some extent. Although the star is widely separated from any companions, so there are no confusion issues, at $V \sim 9$ it is bright for these instruments. The Tycho data are entirely flagged and have large errors so these are unusable. The ROTSE-I instrument provides an unfiltered magnitude close to $R$ every few days and covers the 1999 - 2000 observing season (JD = 2451460 - 2451630) (Akerlof et al., 2000). The data are taken from the NSVS Public Data Release (Woźniak et al., 2004). However, of the 127 observations only 20 points have "good" flags according to the criteria of Wils et al. (2006) while the rest are suspect and/or have large errors. For ROTSE-I the good data show no sign of variation with a mean magnitude, $M_{\mathrm{ROTSE}}=9.487 \pm 0.022(\mathrm{sd})$ with no suspiciously faint points. NSV 4031 was also observed by The Amateur Sky Survey (TASS) (Droege et al., 2006), and although the data are no longer available a measure 
Table 2: Observing log of multiple observations and time series runs

\begin{tabular}{cccccr}
\hline Date & JD & Hours & Instrument & Band & $\mathrm{N}$ \\
\hline \hline 2013 Nov 30 & $2456627.454-.589$ & 3.2 & 2-in Titan & $C V$ & 269 \\
2013 Dec 04 & $2456631.422-.477$ & 1.3 & 2-in Titan & $C V$ & 116 \\
2013 Dec 10 & $2456637.385-.634$ & 6.0 & Shobdon & $V$ & 1528 \\
2013 Dec 28 & $2456655.364-.406$ & 1.0 & 2-in Titan & $C V$ & 49 \\
2014 Jan 04 & $2456662.492-.565$ & 1.7 & 2-in Titan & $C V$ & 196 \\
2014 Jan 07 & $2456664.605-.625$ & 0.5 & BRT & $C V$ & 2 \\
2014 Jan 10 & $2456667.502-.569$ & 1.6 & Shobdon & $V$ & 57 \\
2014 Jan 12 & $2456669.559-.633$ & 1.8 & Shobdon & $V$ & 45 \\
2014 Jan 29 & $2456686.582-.611$ & 0.7 & BRT & $C V$ & 3 \\
2014 Feb 02 & $2456690.536-.667$ & 3.1 & BRT & $C V$ & 2 \\
2014 Feb 05 & $2456693.529-.541$ & 0.3 & BRT & $C V$ & 2 \\
2014 Mar 19 & $2456736.410-.553$ & 3.4 & BRT & $C V$ & 11 \\
2014 Mar 20 & $2456737.422-.466$ & 1.1 & BRT & $C V$ & 11 \\
2014 Apr 03 & $2456751.413-.445$ & 0.8 & BRT & $C V$ & 4 \\
2014 Apr 20 & $2456768.380-.398$ & 0.4 & BRT & $C V$ & 3 \\
2014 Apr 26 & $2456774.451-.472$ & 0.5 & BRT & $C V$ & 3 \\
2014 Apr 27 & $2456775.412-.483$ & 1.7 & BRT & $C V$ & 6 \\
2014 May 05 & $2456783.384-.457$ & 1.8 & BRT & $C V$ & 2 \\
\hline
\end{tabular}

of the variability is provided through the Welch \& Stetson (1993), index which is based on the consistency of simultaneous $V$ and $I_{\mathrm{C}}$ images, and is catalogued with the mean magnitudes in the TASS Mark IV patches photometric catalog. Based on limited data, only $18 V I_{\mathrm{C}}$ pairs, $W S=0.41$, which suggests no variation with $V=9.225 \pm 0.013(\mathrm{sd})$ and $I_{\mathrm{C}}=8.711 \pm 0.038(\mathrm{sd})$. Finally, the star was observed by the INTEGRAL Optical Monitoring Camera (OMC) (see Alfonso-Garzón et al., 2014, for details) in two short runs, one in 2014 and another a year later. NSV 4031 is close to the saturation limit for the OMC but it gives consistent magnitudes, $V=9.19 \pm 0.04$ and $9.24 \pm 0.05$, with no sign of variation during the runs.

SuperWASP (Pollacco et al., 2006; Butters et al., 2010) data are taken in long runs of typically several hours per night as available over two or three observing seasons. The usable magnitude range is given as $7 \leq V \leq 15$ but Smalley et al. (2014) limited their selection of stars to $V>8$ to avoid saturation issues, so this is unlikely to be a problem for NSV 4031. However there are other issues. To try to ensure the best selection of data the SuperWASP observations have been taken from the longer time-series runs and have been limited to data with an external consistency, mean $\sigma<0$. 03 , averaged over several consecutive points as opposed to the internal uncertainties attached to the individual observations. The bulk of the data cover the $2007-2008$ observing season (JD $=2454400$ - 2454560), although there is one run from the previous season.

The longest run of data is taken from the All-Sky Automated Survey for Supernovae (ASAS-SN) (Shappee et al., 2014; Kochanek et al., 2017) which typically provides $1-3$ $V$ or $g$-band magnitudes every few days throughout the observing season. Observations are available from 2012 to the present. Since 2018 the project has been increasingly 


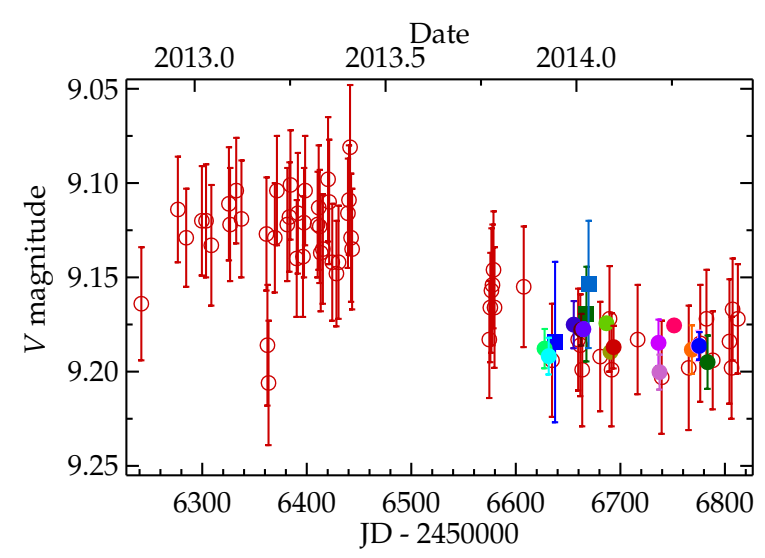

Figure 1: Epoch plot of the new data showing the single observations (open circles) and means of the multiple and time-series observations (filled symbols) with different runs shown in different colours.

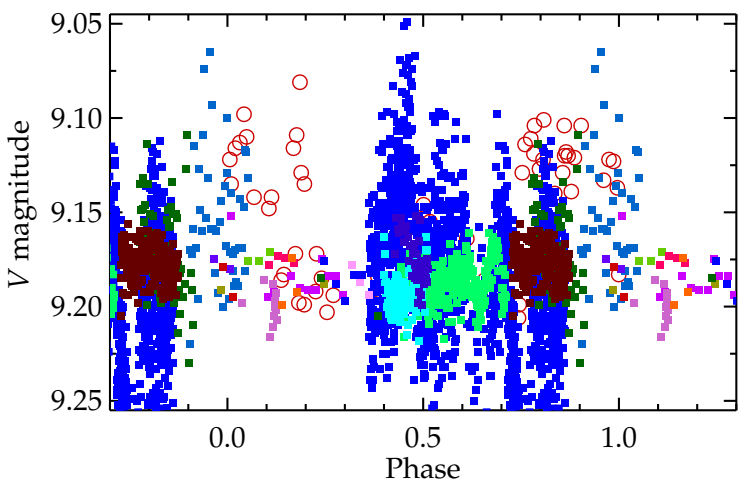

Figure 2: Phase diagram of the new data folded on Lange's ephemeris. The symbols are as before but all the individual observations are shown. Some of these time-series points fall outside the plot.

using the SDSS $g$ filter, but only the $V$ band data are shown here. The saturation limit for the ASAS-SN instruments is $V \sim 10-11$ but the image processing employs a correction procedure which aims to provide useful data from saturated images. How well the correction works depends on many factors but it can fail and produce spurious data which may present in various ways (see Kochanek et al., 2017, for details). NSV 4031 is a magnitude brighter than the saturation limit and it is clear that some of the data are not well corrected, but it all has to be affected in some way.

\section{Results}

The light curve of the data from 2012 - 2014 observing seasons is shown in Figure 1 and the step in the BRT data between the two seasons is obvious. Nevertheless the scatter in the data is consistent with the uncertainties and there are no deep eclipses. When folded on Lange's ephemeris (see Figure 2) there is also no indication of an eclipse and the phase diagram is relatively well covered, although there is considerable scatter for the reasons given earlier. A Discrete Fourier Transform (DFT) periodogram of the data shows a very noisy and broad peak near 3 cycles $\mathrm{d}^{-1}$ with a full amplitude of $0 . \mathrm{m} 04$, which is consistent with the scatter in the data.

The SuperWASP data provide 37 time series with more than 20 points and those from the second season are shown in Figure 3. The mean of the data is $V=9.186 \pm 0.028(\mathrm{sd})$ which is consistent with the scatter of the individual runs and the noise limit on the selected data. Figure 4 shows the data folded on Lange's ephemeris and reveals two important points. The first is that many of the runs show trends of $\sim 0.05$ on a semidiurnal time scale, which are probably extinction related. The second point is that the phase coverage is not complete so although Lange's phase zero has been observed any putative eclipse could have migrated to the unobserved part of the phase diagram, unlikely as this may be. However, in these data there is no suggestion of an eclipse. A DFT 


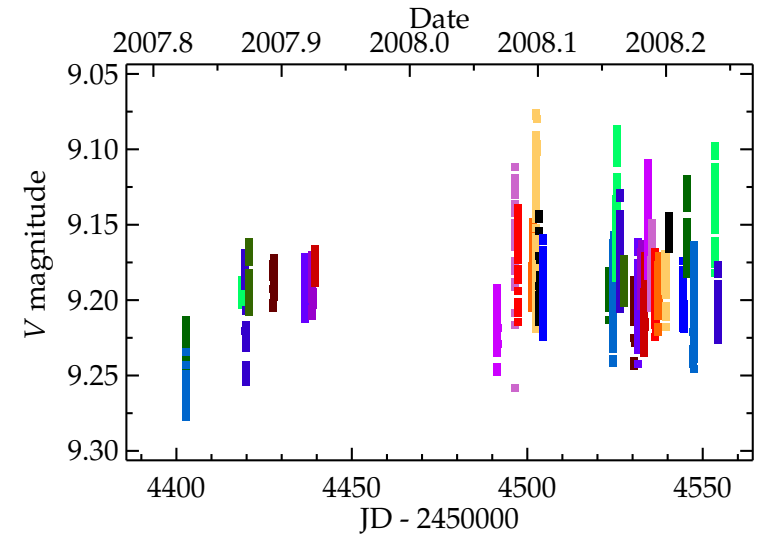

Figure 3: Epoch plot of the selected SuperWASP runs from the second season. Different runs are identified by different colours.

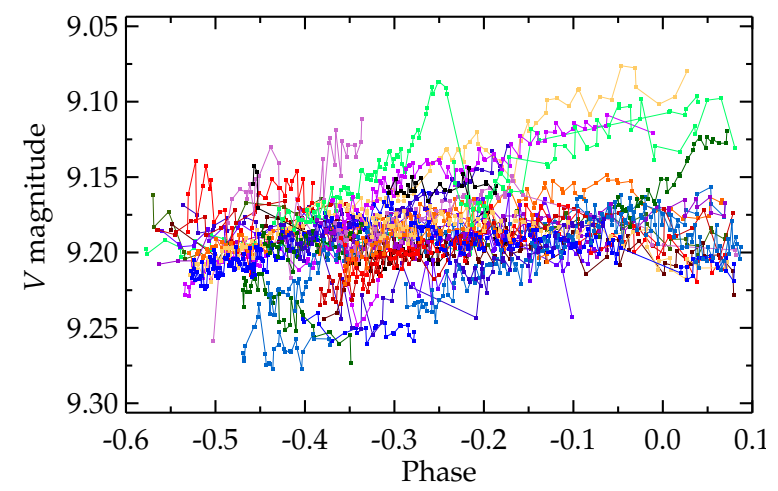

Figure 4: Phase diagram of the selected SuperWASP runs folded on Lange's ephemeris. The colours are as before.

periodogram of the data shows a peak near 2 cycles $\mathrm{d}^{-1}$ with a full amplitude of 0 . 03 . Reassuringly this result is consistent with Figure 4, but it is barely above the noise level so any periodic signal must be below this limit.

The other large data set is provided by ASAS-SN but this is compromised by varying levels of saturation. The epoch plot in Figure 5 shows all the $V$-band data from 2012 to the present. The bulk of the data lie at $V \sim 9.4$ but there is an additional separate band at $V \sim 9.2$ and a scatter of points fainter than $V \sim 9.6$. Poorly corrected saturation tends to produce erroneously faint points but it can also produce bright points which seem to be the majority here. However, the main band also has significantly larger scatter than would be expected with a mean of $V=9.400 \pm 0.051$. The DFT periodogram of the

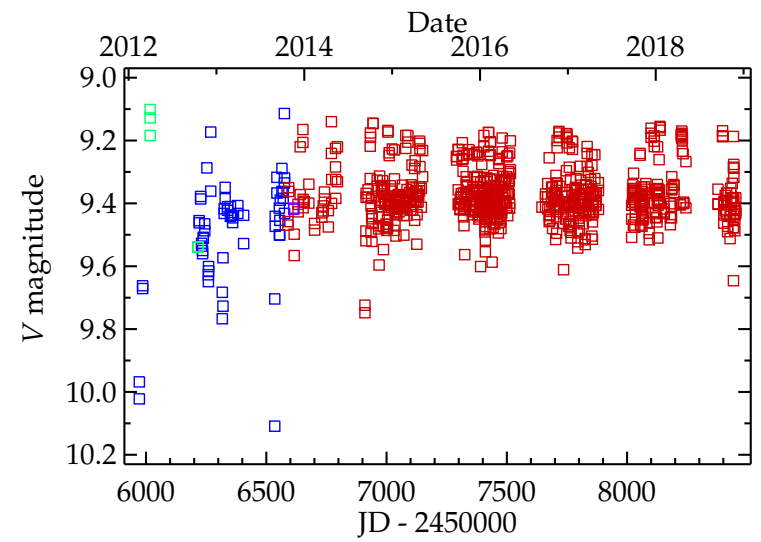

Figure 5: Epoch plot of the ASAS-SN $V$-band data showing the broad central band and the narrow strip of brighter points, and the scatter of faint points. Different cameras are shown in different colours.

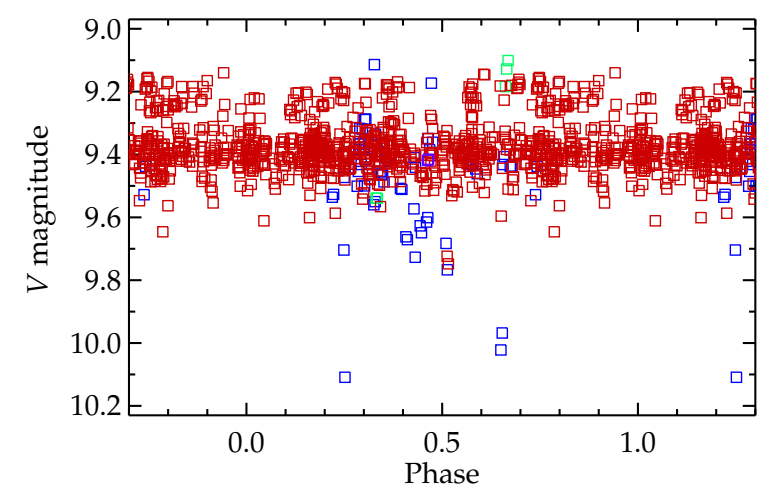

Figure 6: Phase diagram of the ASAS-SN data folded on Lange's ephemeris. There is no obvious periodicity to the faint points. 
main block of data also shows a peak near 2 cycles $\mathrm{d}^{-1}$, similar to the SuperWASP data, but with a full amplitude of 0 . 05 , and again this is probably an hour-angle effect. The main peak with its 1 -day aliases lie significantly above the noise level, $\sim 0.03$, so in that sense it is real, but while it is present in the data it is probably not present in the star. The phase diagram of the ASAS-SN $V$-band data folded on Lange's ephemeris is shown in Figure 6. The whole cycle is well covered and there is no indication of an eclipse, nor any coherent faint points. The ASAS-SN $g$-band data covering the past three observing seasons show very similar saturation effects, and with a clearer diurnal variation.

\section{Luminosity and Spectral Energy Distribution}

The position of NSV 4031 places it at $35^{\circ}$ above the galactic plane so extinction will be relatively low, and Gaia is able to provide a remarkably precise value for the distance of $230 \pm 3$ pc (Gaia Collaboration et al., 2018; Bailer-Jones et al., 2018) The NASA/IPAC Infrared Science Archive gives the total reddening in this direction as $E_{B-V}=0.04-0.05$ (Schlegel et al., 1998; Schlafly \& Finkbeiner, 2011) while Gontcharov \& Mosenkov (2017) give $E_{B-V}=0.06$ for NSV 4031. By contrast Green et al. (2019) suggest that all the reddening occurs between 200 and $240 \mathrm{pc}$, so for NSV $4031 E_{g-r}=0.02 \pm 0.02$. Taking $R_{V}=3.1$ this gives $A_{V} \sim 0.05-0.2$, but probably nearer the lower end of the range. When combined with the distance and magnitude, taken as $V=9.2$, this yields absolute magnitudes of $M_{V}=2.4$ with no reddening and obviously $M_{V}=2.2$ with $A_{V}=0.2$. Using the Rochester calibration this corresponds to a late A-type main sequence star (see Pecaut \& Mamajek, 2013, for details).

The widely quoted spectral type of NSV 4031 is G5 but this comes originally from the HD catalogue. More recently Ofek (2008) and Pickles \& Depagne (2010) have derived a spectral type of F5 IV by fitting photometry from a number of optical and near-infrared bands to spectral templates. The same process is repeated here using all the available

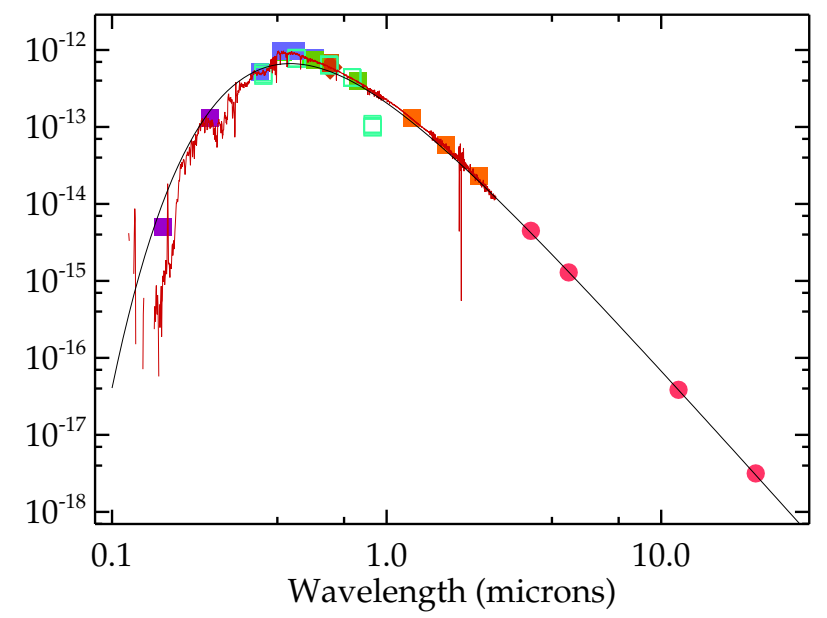

Figure 7: Spectral energy distribution of NSV 4031 from the photometric sources given in the text. The red line is the best fit spectrum and the black line is the best fit black-body curve of $6500 \mathrm{~K}$. 
photometric data to construct the best current spectral energy distribution which is shown in Figure 7. Magnitudes have been taken from Galex FUV and NUV (Bianchi et al., 2011), Stromgren uvby (Hauck \& Mermilliod, 1998), Tycho $B_{\mathrm{T}} V_{\mathrm{T}}$ (Høg et al., 2000), Carlsberg Meridian Catalogue $15 r^{\prime}$, (Niels Bohr Institute et al., 2014), SDSS Photometric Catalogue, Release 12 ugriz (Alam et al., 2015), TASS Johnson-Cousins $V I_{\mathrm{C}}$ (Droege et al., 2006), Gaia DR2 G, BP, RP (Gaia Collaboration et al., 2018), 2MASS JHK (Cutri et al., 2003; Skrutskie et al., 2006) and WISE $W 1, W 2, W 3$, and $W 4$ (Wright et al., 2010; Cutri \& et al., 2014).

The SED has been compared with spectra from the Pickles (1998) Stellar Flux Library and the best fit is with F5 IV, which is in agreement with Ofek (2008) and Pickles \& Depagne (2010). The distinction between luminosity class V and IV is clear and most marked at the shorter wavelengths. The best fit spectrum is plotted with the data in Figure 7 together with a black-body curve for $6500 \mathrm{~K}$. The small amount of reddening makes very little difference to the fit. Returning to the absolute magnitude the Rochester calibration gives $M_{V}=3.4$ for a main sequence star of spectral type F5, which places NSV 4031 approximately a magnitude above this and is consistent with the F5 IV classification.

\section{Conclusion}

NSV 4031 has not been found to show any variation although the data used suffer from saturation problems, hour angle/extinction issues, extraneous sources of scatter and variations in calibration. Despite these issues there are no consistently faint points that could be interpreted as an eclipse in any of the data sets. Period searches have failed to find any consistent periodic variation above a limit of perhaps 0.05 total amplitude. Most of the variation that has been found can be attributed to hour angle or extinction effects so the true limit of any intrinsic variation is lower than this.

The spectral energy distribution is found to be best matched by spectrum of F5 IV, with little reddening, at a temperature near $6500 \mathrm{~K}$, and this is also found to be consistent with its luminosity. NSV 4031 is most likely a single, slightly evolved star lying $\sim 100 \mathrm{pc}$ above the galactic plane, although it does have a faint CPM companion, GSC 03415-01556, at 270 arcsec (Andrews et al., 2017).

Any claim that a star is not variable is inevitably a hostage to fortune - and most stars are variable a some level - but in this case there is no evidence for any variability above a few hundredths of a magnitude, and no evidence of any eclipses. So what are the other options? If the star does eclipse then it is possible that it has a long period and shows infrequent eclipses, but that would contradict Lange's time scale of one day. It is also possible that the star has stopped eclipsing, and there are well observed cases of stars where apsidal motion and precession of the orbital plane has changed the geometry of the eclipse (see e.g., Zasche \& Paschke, 2012; Juryšek et al., 2018). However that requires a multiple system and the evidence to hand suggest that NSV 4031 is single. Limited radial velicity data also show no significant variation (see Jönsson et al., 2020). Also, it has to be said again that there is no compelling evidence that eclipses were ever seen. So, the star is certainly not an eclipsing binary as described, and is probably not variable at all. 
Acknowledgements: The authors are indebted to Dr Georgij Rudnitskij, editor of Astronomicheskii Tsirkulyar, for a copy and vital translation of Lange's paper. The authors also appreciate helpful comments from the referee. The authors acknowledge use of the Bradford Robotic Telescope operated at that time by the University of Bradford, now the Open University Autonomous Robotic Telescope. This paper makes use of the WASP DR1 data as provided by the computing and storage facilities at the CERIT Scientific Cloud, operated by Masaryk University, Czech Republic. This publication makes use of data products from the Two Micron All Sky Survey, which is a joint project of the University of Massachusetts and the Infrared Processing and Analysis Center/California Institute of Technology, funded by the National Aeronautics and Space Administration and the National Science Foundation. This publication makes use of data products from the Wide-field Infrared Survey Explorer, which is a joint project of the University of California, Los Angeles, and the Jet Propulsion Laboratory/California Institute of Technology, funded by the National Aeronautics and Space Administration. The authors are pleased to acknowledge use of NASA's Astrophysics Data System Bibliographic Services. This research has made use of the SIMBAD database and the VizieR catalogue access tool, CDS, Strasbourg, France (DOI: $10.26093 /$ cds/vizier).

\section{References}

Akerlof, C., Amrose, S., Balsano, R., et al., 2000, AJ, 119, 1901

Alam, S., Albareti, F. D., Allende Prieto, C., et al., 2015, ApJ Suppl, 219, 12

Alfonso-Garzón, J., Montesinos, B., Moya, A., et al., 2014, MNRAS, 443, 3022

Andrews, J. J., Chanamé, J., \& Agüeros, M. A., 2017, MNRAS, 472, 675

Bailer-Jones, C. A. L., Rybizki, J., Fouesneau, M., et al., 2018, AJ, 156, 58

Bianchi, L., Herald, J., Efremova, B., et al., 2011, ApछSS, 335, 161

Butters, O. W., West, R. G., Anderson, D. R., et al., 2010, A\&A, 520, L10

Cutri, R. M. \& et al., 2014, VizieR Online Data Catalog, II/328

Cutri, R. M., Skrutskie, M. F., van Dyk, S., et al., 2003, VizieR Online Data Catalog, II $/ 246$

Droege, T. F., Richmond, M. W., Sallman, M. P., et al., 2006, PASP, 118, 1666

Gaia Collaboration, Brown, A. G. A., Vallenari, A., et al., 2018, A\&A, 616, A1

Gontcharov, G. A. \& Mosenkov, A. V., 2017, MNRAS, 472, 3805

Green, G. M., Schlafly, E., Zucker, C., et al., 2019, ApJ, 887, 93

Hauck, B. \& Mermilliod, M., 1998, Ap\&SSS, 129, 431

Høg, E., Fabricius, C., Makarov, V. V., et al., 2000, A $6 A$, 355, L27

Isles, J. E., 1989, J $B A A, \mathbf{9 9}, 14$

Jönsson, H., Holtzman, J. A., Allende Prieto, C., et al., 2020, AJ, 160, 120

Juryšek, J., Zasche, P., Wolf, M., et al., 2018, A\&A, 609, A46

Kochanek, C. S., Shappee, B. J., Stanek, K. Z., et al., 2017, PASP, 129, 104502

Lange, G. A., 1963, Astronomicheskij Tsirkulyar, 246, 3

Lloyd, C., 2018, VSS Circ, 176, 39 
Loughney, D., 2015, BAA Eclipsing Binary Observing Guide, The British Astronomical Association

Niels Bohr Institute, Institute of Astronomy, Cambridge, \& Real Instituto y Observatorio de La Armada, 2014, VizieR Online Data Catalog, I/327

Ofek, E. O., 2008, PASP, 120, 1128

Pecaut, M. J. \& Mamajek, E. E., 2013, ApJ Suppl, 208, 9

Pickles, A. \& Depagne, É., 2010, PASP, 122, 1437

Pickles, A. J., 1998, PASP, 110, 863

Pollacco, D. L., Skillen, I., Collier Cameron, A., et al., 2006, PASP, 118, 1407

Schlafly, E. F. \& Finkbeiner, D. P., 2011, ApJ, 737, 103

Schlegel, D. J., Finkbeiner, D. P., \& Davis, M., 1998, ApJ, 500, 525

Shappee, B. J., Prieto, J. L., Grupe, D., et al., 2014, ApJ, 788, 48

Skrutskie, M. F., Cutri, R. M., Stiening, R., et al., 2006, AJ, 131, 1163

Smalley, B., Southworth, J., Pintado, O. I., et al., 2014, A\&A, 564, A69

Welch, D. L. \& Stetson, P. B., 1993, AJ, 105, 1813

Wils, P., Lloyd, C., \& Bernhard, K., 2006, MNRAS, 368, 1757

Woźniak, P. R., Vestrand, W. T., Akerlof, C. W., et al., 2004, AJ, 127, 2436

Wright, E. L., Eisenhardt, P. R. M., Mainzer, A. K., et al., 2010, AJ, 140, 1868

Zasche, P. \& Paschke, A., 2012, A\&A A, 542, L23 